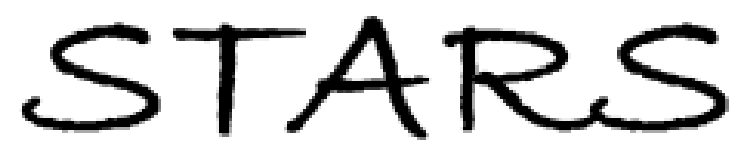

University of Central Florida

STARS

$1-1-2006$

\title{
Modeling of phase volume diffractive gratings, part 1: transmitting sinusoidal uniform gratings
}

Igor V. Ciapurin

University of Central Florida

Leonid B. Glebov

University of Central Florida

Vadim I. Smirnov

Find similar works at: https://stars.library.ucf.edu/facultybib2000

University of Central Florida Libraries http://library.ucf.edu

This Article is brought to you for free and open access by the Faculty Bibliography at STARS. It has been accepted for inclusion in Faculty Bibliography 2000 s by an authorized administrator of STARS. For more information, please contact STARS@ucf.edu.

\section{Recommended Citation}

Ciapurin, Igor V.; Glebov, Leonid B.; and Smirnov, Vadim I., "Modeling of phase volume diffractive gratings, part 1: transmitting sinusoidal uniform gratings" (2006). Faculty Bibliography 2000s. 6035.

https://stars.library.ucf.edu/facultybib2000/6035

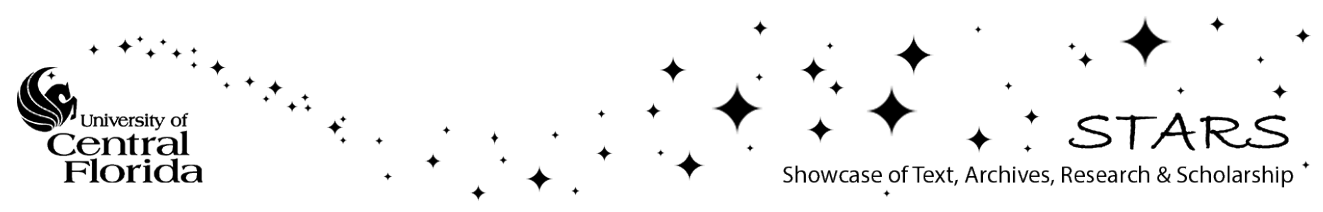




\section{Modeling of phase volume diffractive gratings, part 1: transmitting sinusoidal uniform gratings}

\author{
Igor V. Ciapurin, MEMBER SPIE \\ Leonid B. Glebov, MEMBER SPIE \\ University of Central Florida \\ College of Optics and Photonics/CREOL \& FPCE \\ Center for Research and Education in \\ Optics and Lasers \\ Florida Photonics Center of Excellence \\ P.O. Box 162700 \\ Orlando, Florida 32816-2700 \\ E-mail: igor@creol.ucf.edu
}

\section{Vadim I. Smirnov \\ OptiGrate \\ 3267 Progress Drive}

Orlando, Florida 32826

\begin{abstract}
A detailed model of the diffraction of plane and Gaussian beams on plane uniform phase Bragg gratings based on Kogelnik's theory of coupled waves is presented. The model describes transmitting gratings with arbitrary orientation in a plane-parallel plate taking into account spectral width and angular divergence of laser beams along with material dispersion of a photosensitive medium. The model results are compared with experimental data for high-efficiency Bragg gratings in a photothermorefractive (PTR) glass. () 2006 Society of Photo-Optical Instrumentation Engineers. [DOI: 10.1117/1.2159470]
\end{abstract}

Subject terms: volume holographic gratings; Bragg diffraction; theory of diffraction; numerical approximation and analysis; holographic recording materials.

Paper 040976R received Dec. 18, 2004; revised manuscript received May 25 2005; accepted for publication Jun. 9, 2005; published online Jan. 24, 2006.

\section{Introduction}

Over recent decades, numerous publications have appeared on theoretical and experimental studying of volume Bragg gratings (VBGs) recorded in various phase photosensitive media and used in different configurations. A most widely used basis for description of such gratings is the theory of coupled waves ${ }^{1}$ developed by Kogelnik in 1969. Its results were applied for further theoretical consideration ${ }^{2-5}$ and the treatment of experimental results observed for VBGs in photorefractive crystals, ${ }^{6-10}$ dichromated gelatin, ${ }^{11,12}$ photopolymers, ${ }^{13}$ and inorganic photosensitive glasses. ${ }^{14-16}$ There are several more approaches describing VBGs, e.g., rigorous coupled-wave analysis ${ }^{17}$ and the beampropagation method, ${ }^{18}$ which were compared with coupledwave theory in Refs. 19 and 20. However, Kogelnik's theory is still the most commonly used approach for the volume grating modeling.

Today, VBGs are considered perfect spectral and/or angular selectors with highly adjustable parameters. Angles of incidence and diffraction, central wavelength, and spectral/ angular width can be properly chosen by varying the grating thickness, period of refractive index modulation, and grating vector orientation. VBGs are used for spectral beam combining of high-power laser beams with shifted wavelengths, ${ }^{21-23}$ coupling elements in laser resonators, ${ }^{24-27}$ beam deflectors, splitters, attenuators, etc.

One of the most promising materials for VBGs is a photothermorefractive (PTR) glass, which is a silicate glass doped with silver, cerium, and fluorine. ${ }^{28}$ This glass was successfully used for high-efficiency holographic elements in high-power laser systems. ${ }^{16,29}$ Both transmitting and reflecting PTR VBGs were observed to exhibit diffraction efficiency greater than $95 \%$ as well as perfect thermal, optical, and mechanical stability in high-power beams. This is

0091-3286/2006/\$22.00 @ 2006 SPIE why our modeling is illustrated for those variations of grating parameters that are typical for PTR VBGs: the refractive index is in the range of 1.5 at wavelengths ranging from 0.4 to $2.7 \mu \mathrm{m}$, refractive index modulation is up to $1000 \mathrm{ppm}\left(10^{-3}\right)$, and grating thickness is from 0.2 to $20 \mathrm{~mm}$.

The goal of this work is to reduce Kogelnik's theory to practical formulas that enable practical modeling and design of diffractive optical elements based on VBGs. This part of modeling considers diffraction of plane monochromatic, divergent, and polychromatic laser beams on uniform sinusoidal lossless transmitting volume gratings and compares the model with experimental results in PTR Bragg gratings. Further parts will describe modeling of reflecting volume gratings (holographic mirrors) as well as the application of both transmitting and reflecting grating for spectral beam combining for different types of lasers.

\section{Basic Definitions of Beam Propagation and Diffraction in Bragg Gratings}

Let us consider a plate of a photosensitive material with a volume phase grating. The surface of a plate that is crossed by an incident beam is the front surface. Depending on diffraction angle and orientation of a grating in the plate, one can distinguish several types of Bragg gratings. A grating is called a transmitting Bragg grating if the diffracted beam crosses the back surface; reflecting, if the diffracted beam crosses the front surface; and prismatic, if the diffracted beam crosses one of the side surfaces. Let us describe a plane transmitting Bragg grating recorded in the volume of a photosensitive medium by sinusoidal modulation of a refractive index and occupying the whole volume of a plane-parallel plate, as shown in Fig. 1. This volume grating could be entirely described by the following set of parameters: an average refractive index of a medium $n_{\mathrm{av}}$ at free-space wavelength $\lambda_{0}$; an amplitude of refractive index 


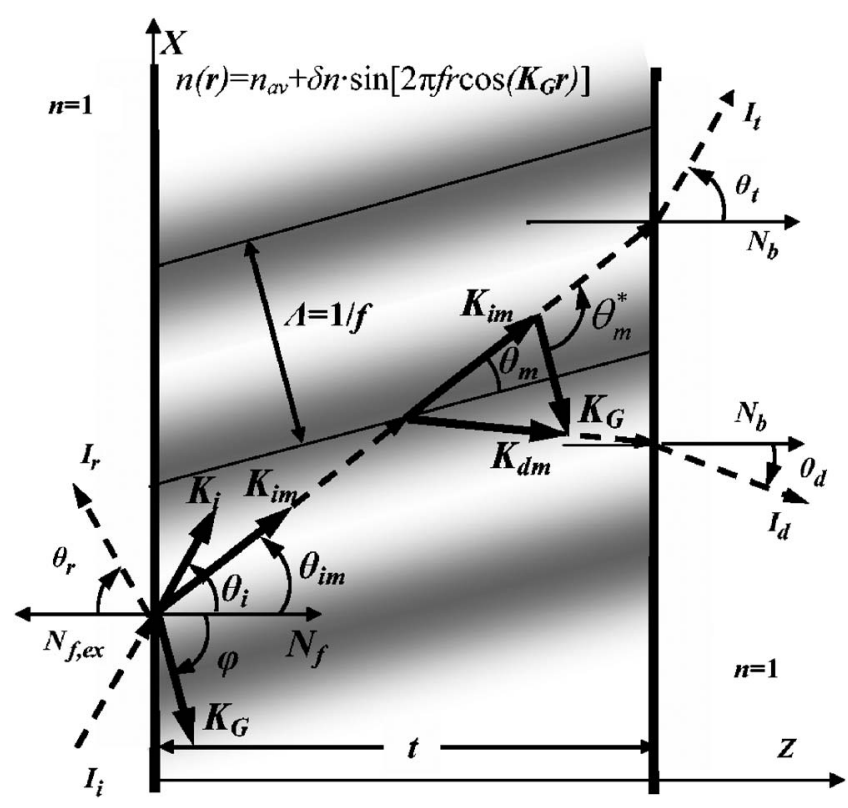

Fig. 1 Propagation of optical rays through a volume Bragg grating: $\boldsymbol{N}_{f}$ and $\boldsymbol{N}_{f, \text { ex }}$, normals to the front surface for incident $\left(I_{i}\right)$ and reflected $\left(I_{r}\right)$ beams; $\boldsymbol{N}_{b}$, normal to the back surface for the transmitted $\left(I_{t}\right)$ and diffracted $\left(I_{d}\right)$ beams; $\boldsymbol{K}_{i}, \boldsymbol{K}_{\mathrm{im}}$, and $\boldsymbol{K}_{\mathrm{dm}}$, wave vectors of incident beam in air, and incident and diffracted beams in the medium; $\boldsymbol{K}_{G}$ grating vector; $\varphi$, grating inclination; $\theta_{i}, \theta_{r}, \theta_{\mathrm{im}}, \theta_{t}$, and $\theta_{d}$, angles of incidence, reflection, incidence in medium, transmission, and diffraction; $\theta_{m}$, Bragg angle; and $\theta_{m}^{*}$, incident Bragg angle.

modulation $\delta n$; the period $\Lambda$ or spatial frequency $f=I / \Lambda$; the grating thickness $t$; and the inclination angle $\varphi$ between the normal to the front surface $\boldsymbol{N}_{f}$ and grating vector $\boldsymbol{K}_{G}$, which is directed toward a medium perpendicular to the planes of a constant refractive index and has a module $\left|\boldsymbol{K}_{G}\right|=2 \pi f$. The lateral size of the grating should not be a parameter while the grating occupies the whole volume of the plate.

Determination of angles in Bragg gratings is similar to those in classical geometrical optics. Figure 1 shows an incident beam $\boldsymbol{I}_{i}$ approaching front surface of the plate at the angle $\theta_{i}$, then is reflected from the front surface at the angle $\theta_{r}$ and refracted into the medium at the angle $\theta_{\mathrm{im}}$. An incident angle $\theta_{i}$ is the angle between the normal to the front surface $\boldsymbol{N}_{f}$ directed toward the incident beam propagation and the wave vector of an incident beam $\boldsymbol{K}_{i}$. It is positive if $\boldsymbol{K}_{i}$ is in a counterclockwise direction from the $N_{f}$, and can vary from $-\pi / 2$ to $+\pi / 2$. A reflection angle $\theta_{r}$ has the same module as $\theta_{i}$ but an opposite sign. An incident angle in medium has the same sign as the incident angle in air and its value is determined according to the Snell's law $\sin \theta_{i}=n_{\mathrm{av}} \sin \theta_{\mathrm{im}}$.

The angle of grating inclination (or tilt, or slant), $\varphi$, is the angle between the normal to the front surface $\boldsymbol{N}_{f}$ for an incident beam and the grating vector $\boldsymbol{K}_{G}$. It is positive in the counterclockwise direction and can vary from $-\pi / 2$ to $+\pi / 2$. For example, Fig. 1 shows the negative inclination of a grating $\varphi<0$. A transmitting grating with inclination of $\varphi= \pm \pi / 2$ is called a symmetric or normal grating.

To describe Bragg diffraction in all types of volume gratings regardless of type and inclination, let us introduce

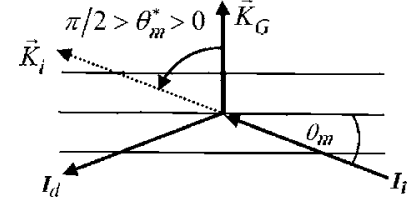

Forward (+) Bragg order

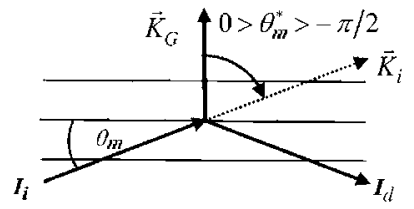

Forward $(-$ ) Bragg order
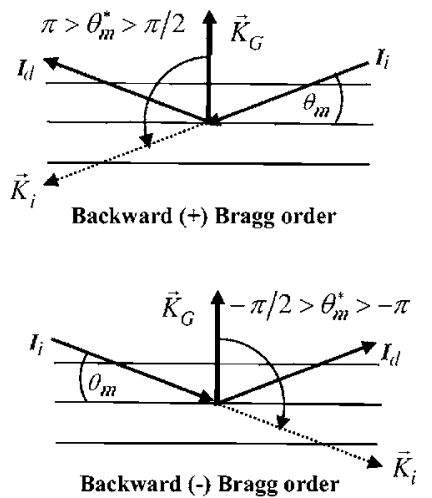

Fig. 2 Possible orders of Bragg diffraction inside medium: $I_{i}$ and $I_{d}$, incident and diffracted beams; $\boldsymbol{K}_{i}$, wave vector of incident beam; $\boldsymbol{K}_{G}$, grating vector; $\theta_{m}$, Bragg angle; $\theta_{m}^{*}$, incident Bragg angle.

an incident Bragg angle in a medium $\theta_{m}^{*}$. This angle is determined as an angle between a grating vector $\boldsymbol{K}_{G}$ and a wave vector $\boldsymbol{K}_{\mathrm{im}}$ of a refracted beam inside the medium, and it can vary from $-\pi$ to $+\pi$. One can distinguish the following possible cases of Bragg diffraction depicted in Fig. 2. The positive orders of Bragg diffraction are for incident Bragg angle ranged from 0 to $+\pi$, i.e., the counterclockwise direction of an incident beam from the grating vector. The negative orders of Bragg diffraction are for incident Bragg angle ranging from 0 to $-\pi$, i.e., the clockwise direction of an incident beam from grating vector. The forward orders of Bragg diffraction are for a module of an incident Bragg angle less than $\pi / 2$. The backward orders of Bragg diffraction are for a module of an incident Bragg angle more than $\pi / 2$. Thus, depending on mutual orientation of grating and incident wave vectors, one can distinguish four Bragg orders, e.g., "plus forward" or "minus backward" etc.

Several examples of transmitting Bragg gratings recorded with different angles of inclination in a planeparallel photosensitive plate are shown in Fig. 3. Figures 3(a) and 3(b) show positive inclination angles $\varphi>0$. For a positive inclination angle $\varphi$ close to $+\pi / 2$ and positive incident angle $\theta_{i}$ [Fig. 3(a)], an incident Bragg angle $\theta_{m}^{*}$ is negative with module below $\pi / 2$, and exit diffraction angle $\theta_{d}$ is negative. This is a case of negative forward Bragg diffraction. When an incident angle $\theta_{i}$ is changed to the negative side [Fig. 3(b)], an incident Bragg angle $\theta_{m}^{*}$ is negative with module exceeding $\pi / 2$, and diffracted exit angle $\theta_{d}$ is positive. Transmitting Bragg gratings with a negative inclination $\varphi<0$ are shown in Figs. 3(c) and 3(d). A positive incident angle $\theta_{i}$ corresponds to a positive incident Bragg angle $\theta_{m}^{*}$ with module exceeding $\pi / 2$ and a negative exit diffraction angle $\theta_{d}$ [Fig. 3(d)]. This is a positive backward order of diffraction. A negative incident angle $\theta_{i}$ corresponds to a positive incident Bragg angle $\theta_{m}^{*}$ with module below $\pi / 2$ and a positive exit diffraction angle $\theta_{d}$ [Fig. 3(c)]. This is a positive forward order of diffraction. Note that excitation of Bragg diffraction of a transmitting grating with inclinations not far from $\pi / 2$ by scanning the incident angle of the beam produces switching between forward and backward orders, but not between plus and minus orders. Switching between positive and 


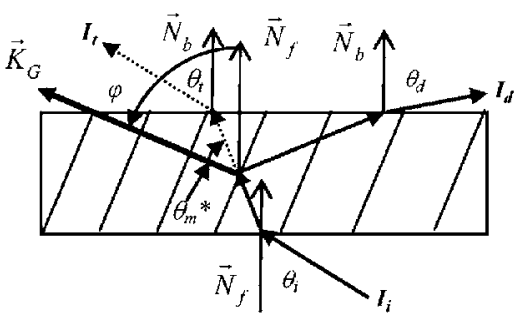

(a) Forward (-) Bragg order

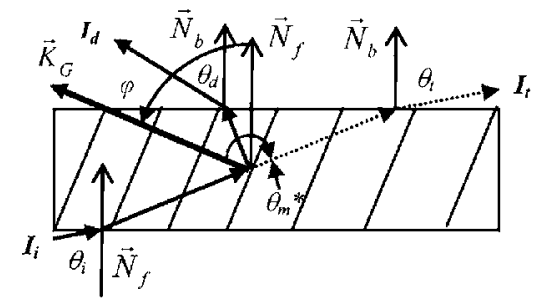

(b) Backward (-) Bragg order

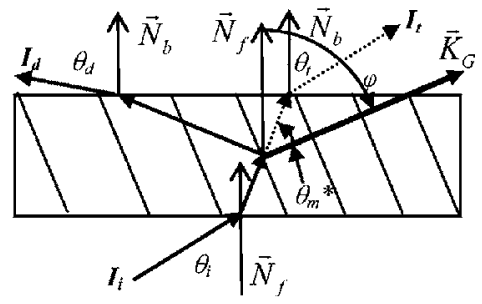

(c) Forward $(+)$ Bragg order

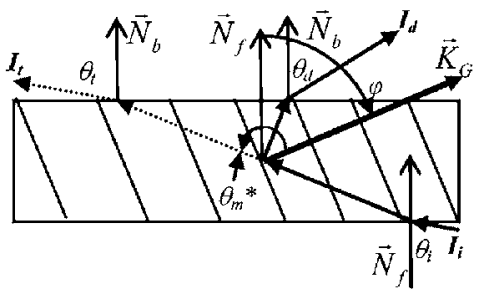

(d) Backward ( + ) Bragg order

Fig. 3 Possible orders of Bragg diffraction for transmitting gratings with different angles of inclination.

negative Bragg orders in a transmitting grating can be observed by changing of illumination from the front surface to the back. For example, Fig. 1 shows a positive backward order of Bragg diffraction for a grating with negative inclination.

The traditionally used (beginning from its crystallography applications) conventional Bragg angle in the media $\theta_{m}$ has been determined as a positive angle not exceeding 90 deg between the plane of a constant refractive index and a direction of the beam propagation. As one can see from Figs. 1 and 2, the relationship between a Bragg angle and an incident Bragg angle is $\sin \theta_{m}=\left|\cos \theta_{m}^{*}\right|$. Note that $\theta_{m}$ does not describe the difference between forward and backward orders of diffraction, which are important for practical modeling of transmitting gratings.

\section{Diffraction of Plane Monochromatic Waves on a Transmitting Bragg Grating}

For volume gratings, a wave vector of a diffracted beam is $\boldsymbol{K}_{\mathrm{dm}}=\boldsymbol{K}_{\mathrm{im}}+\boldsymbol{K}_{G}$ for backward orders and $\boldsymbol{K}_{\mathrm{dm}}=\boldsymbol{K}_{\mathrm{im}}-\boldsymbol{K}_{G}$ for forward orders. Therefore, contrary to the surface grating, diffraction of a beam with a certain wavelength occurs for only one certain angle, which depends on grating spatial frequency according to Bragg's condition:

$\left|\cos \theta_{m}^{*}\right|=\frac{\lambda_{0} f}{2 n_{\mathrm{av}}}$.

In accordance with Kogelnik's theory, ${ }^{1}$ a solution of the scalar wave equation for transmitting VBG gives the following formula for diffraction efficiency (DE):

$\eta=\frac{\sin ^{2}\left(\xi^{2}+\Phi^{2}\right)^{1 / 2}}{1+\xi^{2} / \Phi^{2}}$

Here phase incursion $\Phi$ is the parameter that determines the maximum diffraction efficiency of VBG (grating strength) when the Bragg condition is satisfied, while dephasing parameter $\xi$ describes deviation from the Bragg condition by detuning from either $\theta_{m}^{*}$ or $\lambda_{0}$. Phase incursion in Bragg condition is written in Ref. 1 as

$\Phi=\frac{\pi t \delta n}{\lambda_{0} F_{\varphi}}$,

where parameter $F_{\varphi}$ is an inclination factor:

$F_{\varphi}=\left[-\cos \left(\varphi-\theta_{m}^{*}\right) \cos \left(\varphi+\theta_{m}^{*}\right)\right]^{1 / 2}$.

For normal transmitting gratings with $\varphi= \pm \pi / 2$, the expression for the inclination factor is simplified and becomes

$F_{\pi / 2}=\sin \theta_{m}^{*}=\left[1-\left(\frac{\lambda_{0} f}{2 n_{\mathrm{av}}}\right)^{2}\right]^{1 / 2}$.

The inclination factor describes an additional optical path of incident and diffracted beams in a medium resulting from deviation of propagation from the normal to the surface. Note that this factor is a function of independent Bragg grating parameters because $\theta_{m}^{*}$ must satisfy Bragg condition, Eq. (1).

According to Eq. (2), the DE of a transmitting grating in Bragg condition $(\xi=0)$ is a periodic function of phase incursion $\Phi$ and reaches $100 \%$ when

$\Phi=\pi / 2+j \pi, \quad$ where $j=0,1,2, \ldots$.

Substitution of this phase incursion in Eq. (3) at $j=0$ and considering a Bragg angle value from Eq. (1) gives a minimum thickness of grating $t_{0}$, which provides a $100 \% \mathrm{DE}$ for a given refractive index modulation $\delta n$ :

$t_{0}=\frac{\lambda_{0} F_{\varphi}}{2 \delta n}$.

The dependence of the minimum thickness $t_{0}$ on resonant wavelength $\lambda_{0}$ for normal grating is shown in Fig. 4. For low spatial frequencies, when propagation of incident and diffracted beams are not far from normal to the front sur- 


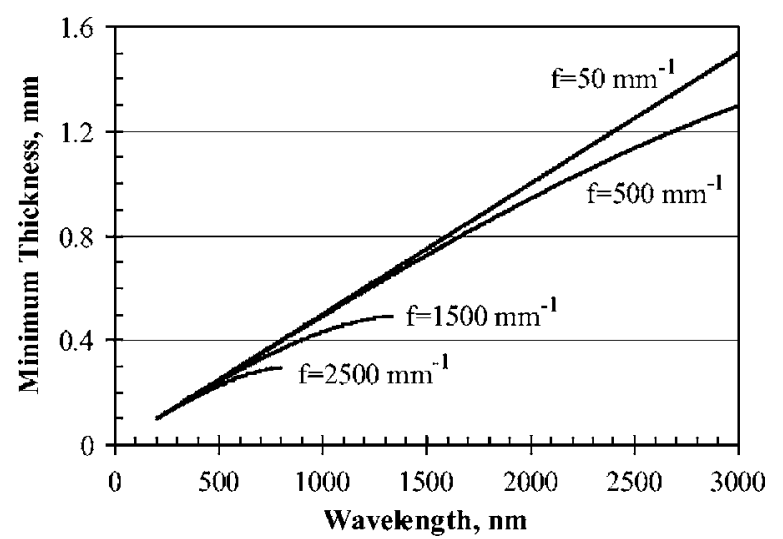

Fig. 4 Dependence of minimum thickness of a Bragg grating that produces $100 \%$ diffraction efficiency at different spatial frequencies on resonant wavelength. Refractive index modulation is $1000 \mathrm{ppm}$.

face, the minimum thickness increases almost linearly with increasing wavelength. This thickness ranges from 0.2 to $1.5 \mathrm{~mm}$ for wavelengths varied from 400 to $3000 \mathrm{~nm}$. However, increasing the spatial frequency of grating results in increasing the optical path of incident and diffracted beams in a plate and, therefore, leads to decreasing the inclination factor and corresponding deviation from the linear dependence. For gratings with a high spatial frequency, radiation with a wavelength exceeding some value cannot be diffracted because the required incident angle in a medium exceeds the angle of total internal reflection.

Dephasing parameter $\xi$ takes into account small angular deviations $\Delta \theta_{m}$ from an incident Bragg angle $\theta_{m}^{*}$ and/or small deviations $\Delta \lambda$ from central wavelength $\lambda_{0}$ :

$$
\xi=\frac{\pi f t}{\cos \left(\varphi-\theta_{m}^{*}\right)-\left(f \lambda_{0} / n_{\mathrm{av}}\right) \cos \varphi}\left(\Delta \theta_{m} \sin \theta_{m}^{*}-\frac{f}{2 n_{\mathrm{av}}} \Delta \lambda\right)
$$

For normal transmitting grating $(\varphi=\pi / 2)$, this expression is simplified and can be written as

$\xi_{\pi / 2}=-\pi f t\left(\Delta \theta_{m}-\frac{f}{2 n_{\mathrm{av}} F_{\pi / 2}} \Delta \lambda\right)$

Because both spectral and angular parts of such detuning from the Bragg condition are equivalent to producing the same value of dephasing parameter $\xi$, interrelation between them could be obtained by equating the terms in parentheses in Eq. (9) and substituting the Bragg condition [Eq. (1)]:

$$
\frac{\Delta \theta_{m}}{\Delta \lambda}=\frac{f}{2 n_{\mathrm{av}} F_{\pi / 2}}
$$

Equation (9) is a universal interrelation between spectral and angular selectivity of a VBG that enables easy calculation of one of them from the given (or measured) other.

The angular selectivity of a VBG for a resonant wavelength $\lambda_{0}$ could be determined by substituting Eqs. (3) and (8) into Eq. (2) at $\Delta \lambda=0$. The general formula for angular selectivity is rather cumbersome, but a simplified formula for normal transmitting grating shows all features of this type of gratings:

$$
\eta\left(\Delta \theta_{m}\right)=\frac{\sin ^{2}\left\{\pi t\left[\left(\delta n / \lambda_{0} F_{\pi / 2}\right)^{2}+\left(f \Delta \theta_{m}\right)^{2}\right]^{1 / 2}\right\}}{1+\left(\lambda_{0} f F_{\pi / 2} \Delta \theta_{m} / \delta n\right)^{2}} .
$$

The dependence of diffraction efficiency on detuning from the Bragg angle is shown in Fig. 5(a). Curve 1 corresponds to 2-mm-thick VBG with a $1086-\mathrm{mm}^{-1}$ spatial frequency and a 250 -ppm refractive index modulation, which provides $100 \%$ diffraction efficiency at $1085 \mathrm{~nm}$. One can see a well-known central maximum and a number of sidelobes with gradually decreasing magnitude. Curve 2 shows a decrease of DE resulting from a decrease of refractive index modulation down to $125 \mathrm{ppm}$ at the same grating thickness and spatial frequency; this decreases $\mathrm{DE}$ at the central maximum down to $50 \%$, but positions of minima and maxima of the sidelobes practically are not changed. Curve 3 shows a decrease of DE resulting from a decrease of the thickness down to $1 \mathrm{~mm}$ for the same $\delta n=250 \mathrm{ppm}$; this also provides DE of $50 \%$, but it causes dramatic widening of angular selectivity, when the first minimum moves to the position of the second minimum for 2-mm-thick gratings.

Note that Eq. (2) requires the following criterion to equalize the DE to zero:

$\left(\xi^{2}+\Phi^{2}\right)^{1 / 2}=j \pi, \quad$ where $j=1,2, \ldots, \mathrm{n}, \ldots$.

Let us determine angular selectivity inside the VBG medium at the HWFZ level, $\delta \theta_{m}^{\mathrm{HWFZ}}$, as the angle between the central maximum and the first minimum on the DE curve. For VBGs with $100 \%$ diffraction efficiency $\Phi=\pi / 2$. Comparison of Eqs. (9) at $\Delta \lambda=0$ and (12) at $j=1$ gives the following expression for the HWFZ angular selectivity:

$\delta \theta_{m}^{\mathrm{HWFZ}}=\frac{\sqrt{3}}{2 f t_{0}} \approx \frac{0.87}{f t_{0}}$.

Note that the HWFZ angular selectivity $\delta \theta_{m}^{\mathrm{HWFZ}}$ is slightly lower than the widely used grating parameter of FWHM angular selectivity, which for a $100 \%$ efficient grating could be easily estimated as $\delta \theta_{m}^{\mathrm{FWHM}} \approx 1 / f t_{0}$.

Figure 5(b) shows that the angular selectivity of a transmitting VBG with parameters typical for PTR glass could be varied from more than $100 \mathrm{mrad}$ for thin low-frequency gratings to less than $0.1 \mathrm{mrad}$ for thick high-frequency ones. Note that the value of refractive index modulation $\delta n$ providing $100 \%$ DE depends on spatial frequency of grating because different incident angles and should be optimized in accordance with Eq. (7). The angular selectivity of grating in air, $\delta \theta_{i}^{\mathrm{HWFZ}}$, can be easily calculated from Eq. (13) by the use of differential form of Snell's law:

$$
\delta \theta_{i}^{\mathrm{HWFZ}}=\delta \theta_{m}^{\mathrm{HWFZ}}\left(\frac{4 n_{\mathrm{av}}^{2}-\lambda_{0}^{2} f^{2}}{4-\lambda_{0}^{2} f^{2}}\right)^{1 / 2} .
$$

The angular selectivity in air is wider than that in a medium. Their ratio increases from the $n_{\mathrm{av}}$ at low spatial frequencies to about 2.4 at spatial frequency of $1600 \mathrm{~mm}^{-1}$ (see Fig. 6). 

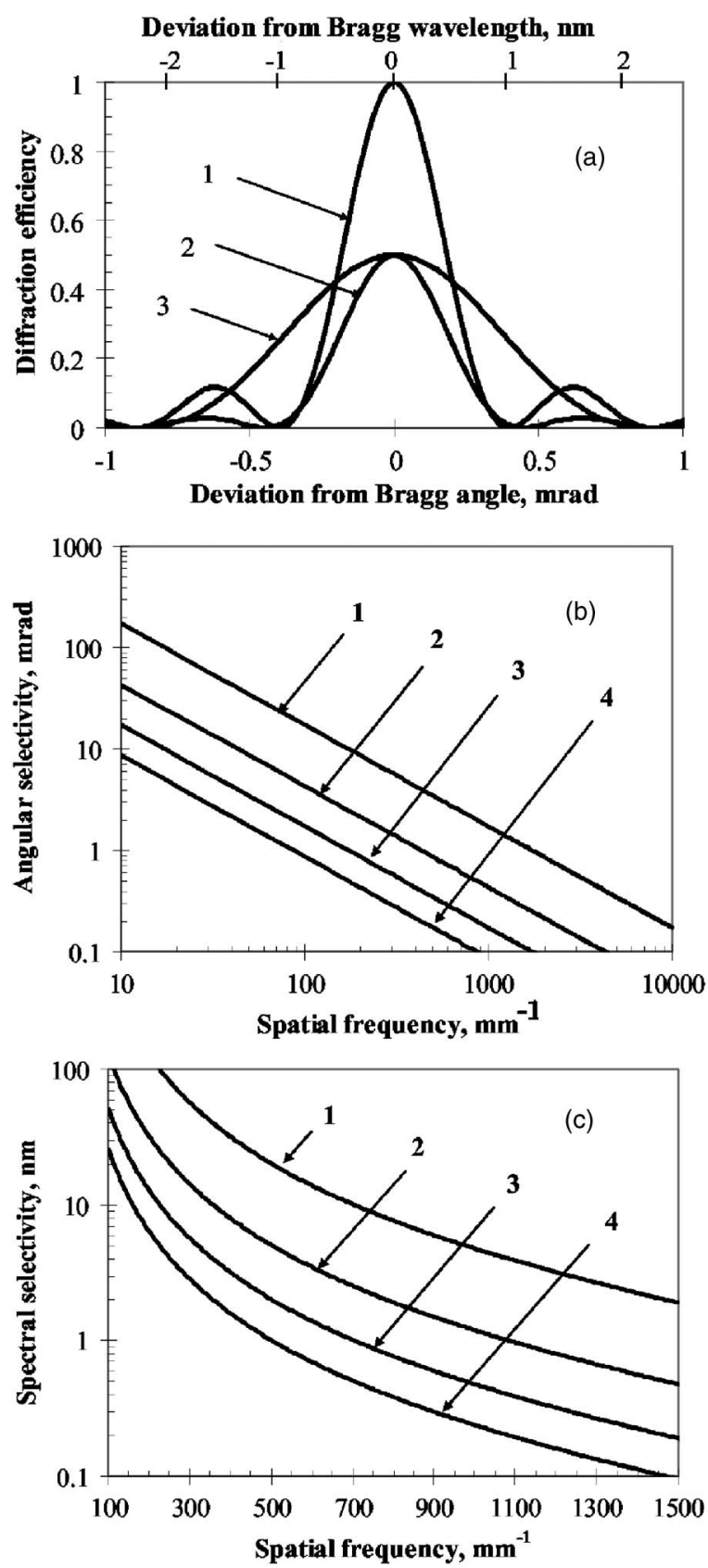

Fig. 5 Selectivity of transmitting Bragg gratings for $\lambda_{0}=1085 \mathrm{~nm}$, $n_{\mathrm{av}}=1.4867$ : (a) dependence of diffraction efficiency on deviation from Bragg angle and wavelength with grating thickness in millimeters: 2.0 for curves 1 and 2 and 1.0 for curve 3; refractive index modulation in parts per million: 250 for curves 1 and 3 and 125 for curve 2; spatial frequency $1086 \mathrm{~mm}^{-1}$; (b) dependence of angular selectivity [half width at first zero (HWFZ)] on spatial frequency for optimal refractive index modulation with grating thickness in millimeters: curve 1, 0.5; curve 2, 2.0; curve 3, 5.0; and curve 4, 10; and (c) dependence of spectral selectivity (HWFZ) on spatial frequency for optimal refractive index modulation with grating thickness in millimeters: curve 1, 0.5; curve 2, 2.0; curve 3, 5.0; and curve 4,10.

In the same manner as already described above for angular selectivity, the $\delta \lambda^{\mathrm{HWFZ}}$ spectral selectivity is determined as a distance between the central maximum and the first zero in spectral distribution of the DE, which could be expressed by substitution of Eqs. (3) and (8) into Eq. (2) at

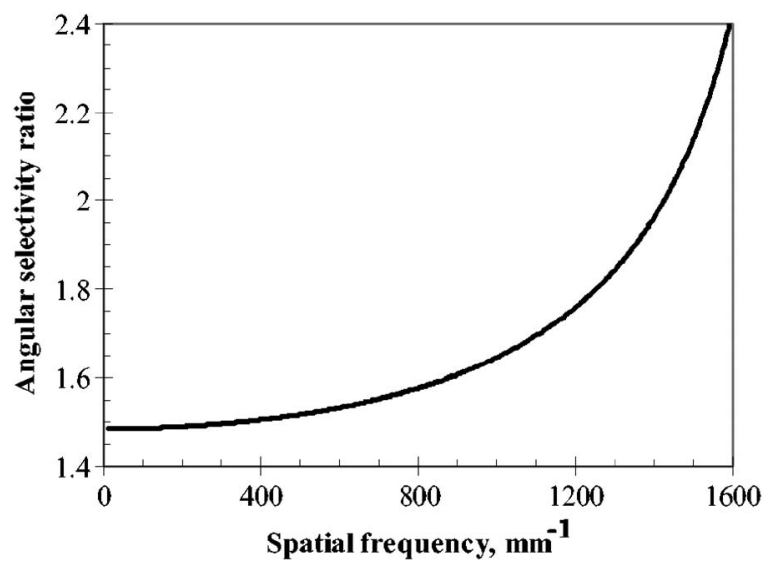

Fig. 6 Ratio of angular selectivity (HWFZ) of normal transmitting VBG in air to that inside a medium for $\lambda_{0}=1085 \mathrm{~nm}$ and $n_{\mathrm{av}}$ $=1.4867$.

$\Delta \theta_{m}=0$. In the case of a normal transmitting grating, this expression is simplified by the use of Eq. (9):

$\eta(\Delta \lambda)=\frac{\sin ^{2}\left\{\frac{\pi t}{F_{\pi / 2}}\left[\left(\delta n / \lambda_{0}\right)^{2}+\left(f^{2} \Delta \lambda / 2 n_{\mathrm{av}}\right)^{2}\right]^{1 / 2}\right\}}{1+\left(f^{2} \lambda_{0} \Delta \lambda / 2 n_{\mathrm{av}} \delta n\right)^{2}}$.

Spectral selectivity [Eq. (15)] has the same structure as angular selectivity [Eq. (11)] because of their linear interrelationship [Eq. (10)]. For the grating parameters depicted in Fig. 5(a), this ratio is $\Delta \lambda / \Delta \theta \cong 500 \mathrm{~mm}^{-1}$. In addition of showing the angular selectivity of 2- and 1-mm-thick transmitting VBGs, Fig. 5(a) shows spectral selectivity of the same gratings, which is represented by the upper horizontal axis of this figure. One can see that all features of spectral selectivity are the same as those already discussed for angular selectivity.

For normal transmitting gratings with $100 \% \mathrm{DE}, \delta \lambda^{\mathrm{HWFZ}}$ could be derived by substitution of Eq. (13) into Eq. (10):

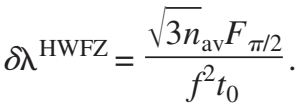

Figure 5(c) shows dependence of spectral selectivity on spatial frequency for different grating thicknesses. The HWFZ spectral selectivity could be easy varied from values below 0.1 to more than $100 \mathrm{~nm}$ by proper choice of grating parameters.

There is an additional mismatching factor from the Bragg condition for beams with different wavelengths due to spectral dispersion of a refractive index in a photosensitive medium. This means that two beams with the same incident and output angles and different wavelengths have different angles of refraction inside the medium, and this leads to the difference in incident Bragg angles. Let us write down the Bragg condition [Eq. (1)], where the refractive index is supposed to depend on wavelength, in a differential form: 


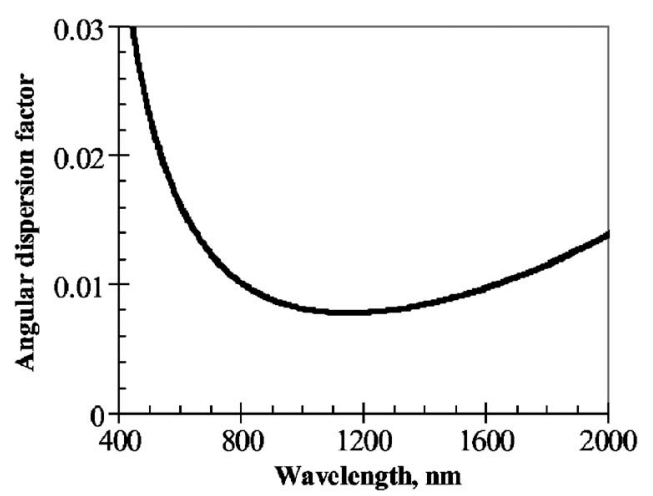

Fig. 7 Effect of material dispersion on the Bragg angle inside a grating medium. The angular dispersion factor is a partial derivative of the Bragg angle over the wavelength resulting from material dispersion of refractive index normalized to absolute value of Bragg angle. The refractive index and material dispersion are determined conform to Ref. 30.

$\frac{\mathrm{d} \theta_{m}}{\mathrm{~d} \lambda}=\left[\frac{1}{\lambda_{0}}-\frac{\mathrm{d} n_{\mathrm{av}} / \mathrm{d} \lambda}{n_{\mathrm{av}}}\right] \cot \theta_{m}^{*}$

In this case, substitution of Eq. (1) into Eq. (17) gives a relative shift of Bragg angle in the medium resulted from material's dispersion:

$\frac{\Delta \theta_{m, \lambda}-\Delta \theta_{m}}{\Delta \theta_{m}}=\frac{\lambda_{0}}{n_{\mathrm{av}}} \mathrm{d} n_{\mathrm{av}} / \mathrm{d} \lambda$.

Estimation of this value for PTR glass, which is classified as crown-type optical glass having $\mathrm{d} n_{\mathrm{av}} / \mathrm{d} \lambda$ ranged $^{30}$ from -10 to $-100 \mathrm{ppm} / \mathrm{nm}$ in the spectral range from near IR to near UV. Using dispersion curve for PTR glass presented in Ref. 30, Eq. (18) gives relative impact of material dispersion on Bragg angle in the medium presented in Fig. 7. One can see that amendment for the Bragg angle does not exceed a few of percent. This means that impact of the material dispersion of a photosensitive medium should be carefully considered for beams with extremely low divergence. For example, by taking into account the material dispersion of PTR glass in the spectral region near $1 \mu \mathrm{m}$, the VBG with an incident Bragg angle of 60 deg would be detuned for $40 \mu \mathrm{rad}$ by shifting of the beam wavelength for $10 \mathrm{~nm}$. This value corresponds to diffraction-limited angular divergence of a beam with 30-mm-aperture and results in completely detuning of high-aperture beam from the Bragg condition.

\section{Diffraction of Gaussian Beams on a Transmitting Bragg Grating}

\subsection{Divergent Monochromatic Beams}

In this section we present the results of Bragg diffraction modeling of a monochromatic beam that has a divergence that could be approximated by a Gaussian function. If the direction of the beam propagation matches the Bragg condition, the normalized function of the beam intensity in the angular space could be written as
$G_{1}(\theta, b)=\exp \left[-2\left(\frac{\theta-\theta_{m}}{b}\right)^{2}\right]$

For a diffraction-limited beam with diameter $D$ at the level of $e^{-2}$ [half width at $\mathrm{e}^{-2}$ of the maximum $\left.\left(\mathrm{HW}^{-2} \mathrm{M}\right)\right]$ at central wavelength $\lambda_{0}$, its divergence $b$ could be determined as

$b=\frac{2 \lambda_{0}}{\pi D}$.

To determine the $\mathrm{DE}$ of a Bragg grating for such a divergent beam, convolution in the angular space of the functions given by Eqs. (15) and (19) should be applied:

$\eta_{\theta}(b)=\frac{\int \eta(\theta) G_{1}(\theta, b) \mathrm{d} \theta}{\int G_{1}(\theta, b) \mathrm{d} \theta}$.

After substitution of the numerical value of a Gaussianfunction integral, Eq. (21) could be written as

$\eta_{\theta}(b)=\sqrt{\frac{2}{\pi}} \frac{1}{b} \int \eta(\theta) G_{1}(\theta, b) \mathrm{d} \theta$.

Figure 8(a) shows angular selectivity of the grating with $\delta \theta_{m}^{\mathrm{HWFZ}}=0.4 \mathrm{mrad}$ and $100 \% \mathrm{DE}$ for a plane monochromatic wave at $1085 \mathrm{~nm}$ for four beams with different divergences $b$. While the beam divergence is much less than the grating angular selectivity (curve 1 corresponds to $b$ $=0.04 \mathrm{mrad}$ ), there is a negligible decrease of diffraction efficiency compare to that for planar wave, and the curve minima reach zero values as it appears for the planar wave [see Fig. 5(a)]. However, if the beam divergence becomes comparable with the grating selectivity, dramatic decreasing of maximal DE occurs (curves 2 to 4 ). When divergence and selectivity values are equal, $b=\delta \theta_{m}^{\mathrm{HWFZ}}$, maximum DE is only about $60 \%$. Also, sidelobes are flattened while the divergence increases, local minima of angular selectivity begins to differ from zero significantly, and at $b \cong \delta \theta_{m}^{\mathrm{HWFZ}}=0.4 \mathrm{mrad}$ the selectivity curve has no local minima at all.

Figure 8(b) shows the dependence of diffraction efficiency on the beam divergence. Four gratings with thicknesses of 20 and $2.0 \mathrm{~mm}$ and a spatial frequency of $357 \mathrm{~mm}^{-1}$ as well as with thickness 2.0 and $0.2 \mathrm{~mm}$ and a spatial frequency $1086 \mathrm{~mm}^{-1}$ have respective values of HWFZ angular selectivity of $0.12,1.2,0.4$, and $4 \mathrm{mrad}$ in accordance with Eq. (13). It was found that diffraction of a divergent beam causes a decrease of DE to $99 \%$ when the beam divergence $b$ becomes 8 times less than the grating HWFZ angular selectivity $\delta \theta_{m}^{\mathrm{HWFZ}}$, i.e., losses are less than $1 \%$ when $8 b \leqslant \delta \theta_{m}^{\mathrm{HWFZ}}$. Further increases of the beam divergence $b$ (e.g., by decreasing of the beam diameter for diffraction-limited beams) results in dramatic decreases of the $\mathrm{DE}$ value. When the beam divergence is equal to the grating angular selectivity $\delta \theta_{m}^{\mathrm{HWFZ}}$, DE decreases almost twice (to $58 \%$ ). 

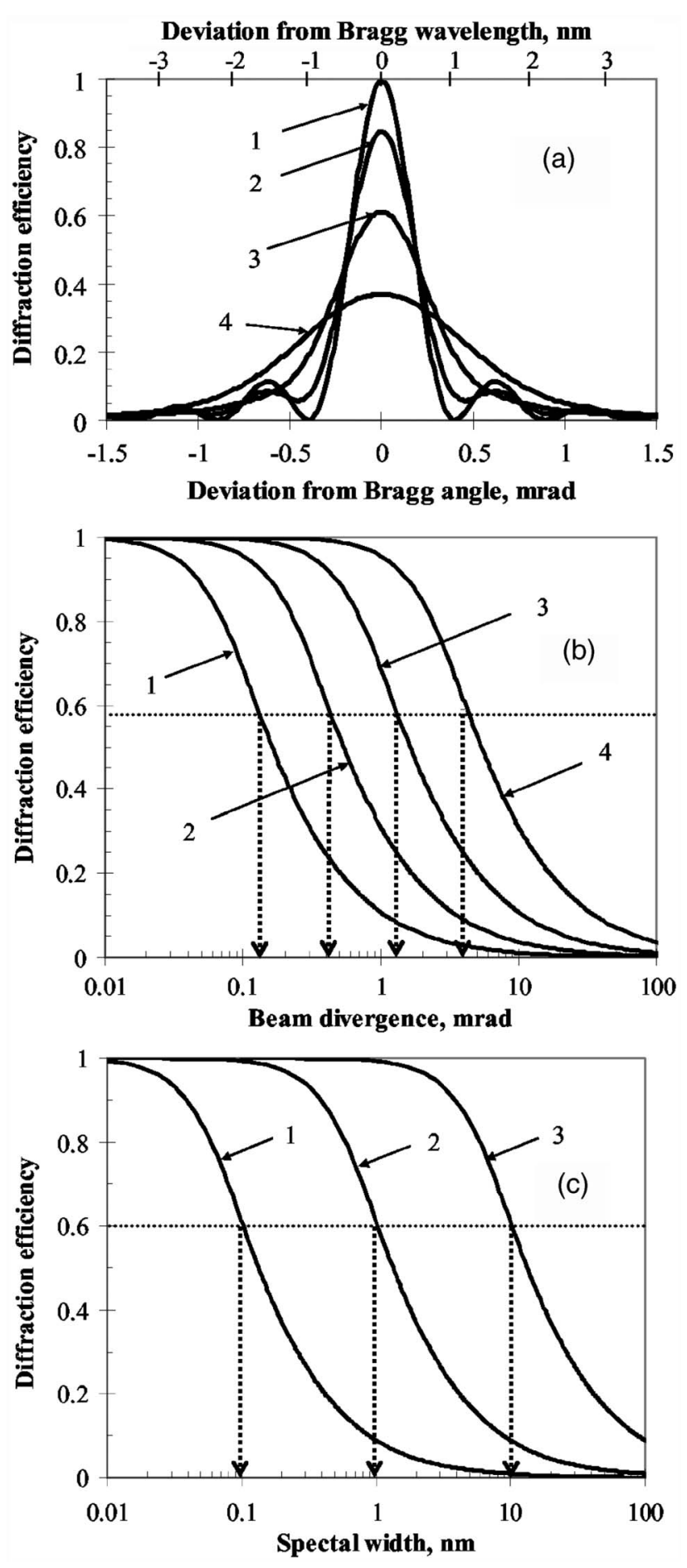

Fig. 8 Selectivity of transmitting VBG for divergent polychromatic beams with central wavelength at $1085 \mathrm{~nm}$ : (a) dependence of DE on detuning from Bragg condition, for a monochromatic wave, DE is $100 \%$; incident beam divergence $\mathrm{HWe}^{-2} \mathrm{M}$ in milliradians: curve 1, 0.04 ; curve 2, 0.2; curve 3,0.4; and curve 4, 0.8; grating angular selectivity is $0.4 \mathrm{mrad}$; beam $\mathrm{HWe}^{-2} \mathrm{M}$ spectral width in nanometers: curve 1, 0.1; curve 2, 0.5; curve 3, 1.0; and curve 4, 2.0; grating spectral selectivity is $1.0 \mathrm{~nm}$; $(C)$ dependence of grating $D E$ on the beam divergence for angular selectivity (HWFZ) of grating in milliradians: curve 1, 0.12; curve 2, 0.4; curve 3, 1.2; and curve 4, 4.0; shown by dotted arrows; and (c) dependence of grating DE on the beam spectral width with spectral selectivity (HWFZ) of grating in nanometers: curve 1, 0.1; curve 2, 1.0; and curve 3, 10; shown by dotted arrows, where the dotted line corresponds to DE for a beam with divergence or spectral width equal to the grating selectivity.

\subsection{Polychromatic Planar Waves}

Let us consider the Bragg diffraction of polychromatic beams with a Gaussian shape of the spectral distribution. The modeling of such diffraction is performed similarly as was done for divergent beams in Sec. 4.1:

$G_{2}(\lambda, w)=\exp \left[-2\left(\frac{\lambda-\lambda_{0}}{w}\right)^{2}\right]$.

The parameter $w$ is the $\mathrm{HW} e^{-2} \mathrm{M}$ spectral width, and $\lambda_{0}$ is a central wavelength of a beam. Diffraction efficiency of transmitting VBG for such beams could be calculated from convolution of the DE for a monochromatic wave determined by Eq. (14) with the Gaussian spectral distribution described by Eq. (23). This gives us the adjusted value of diffraction efficiency $\eta_{\lambda}(w)$ :

$\eta_{\lambda}(w)=\frac{\int \eta(\lambda) G_{2}(\lambda, w) \mathrm{d} \lambda}{\int G_{2}(\lambda, w) \mathrm{d} \lambda}$

Taking into account the numerical value of a Gaussianfunction integral, Eq. (24) could be rewritten similar to Eq. (22):

$\eta_{\lambda}(w)=\sqrt{\frac{2}{\pi}} \frac{1}{w} \int \eta(\lambda) G_{2}(\lambda, w) \mathrm{d} \lambda$.

Equation (25) enables us to calculate the DE of planar polychromatic beams on transmitting VBG. These results are very similar to results described in Sec. 4.1 for the diffraction of divergent beams, and therefore are depicted in the same Fig. 8(a) in the same way as was done for Fig. 5(a). One can see that the beam spectral width is twice fewer than the grating spectral selectivity (curve 2), DE losses about $15 \%$ in the main maximum and gains almost $6 \%$ in each of the first minima. When the beam width is equal to the grating selectivity or exceeds it ( $\left.w \geqslant \delta \lambda^{\mathrm{HWFZ}}\right)$, sidelobes disappear and DE becomes less than $60 \%$.

Figure 8(c) shows the calculated dependence of DE on beam spectral width $w$ for four gratings with the parameters described in Sec. 4.1. One can see that the grating DE is only about $60 \%$ when the beam spectral width $w$ is equal to the grating selectivity $\delta \lambda^{\mathrm{HWFZ}}$. The DE for narrow-spectralline beams is the same as for a monochromatic wave, and it decreases to $99 \%$ when beam spectral width becomes approximately 8 times fewer than the grating HWFZ spectral selectivity, i.e., when $\delta \lambda^{\mathrm{HWFZ}}=8 w$.

\subsection{Divergent Polychromatic Beams: A General Case}

In the case where a real beam is both divergent and spectrally widened, the grating diffraction efficiency is a product of $\eta_{\lambda}(w)$ and $\eta_{\theta}(b)$ calculated in accordance with Eqs. (22) and (25):

$\eta(w, b)=\eta_{\lambda}(w) \eta_{\theta}(b)$.

Thus, both misaligning factors, such as beam divergence and spectral bandwidth, affect the DE of transmitting 


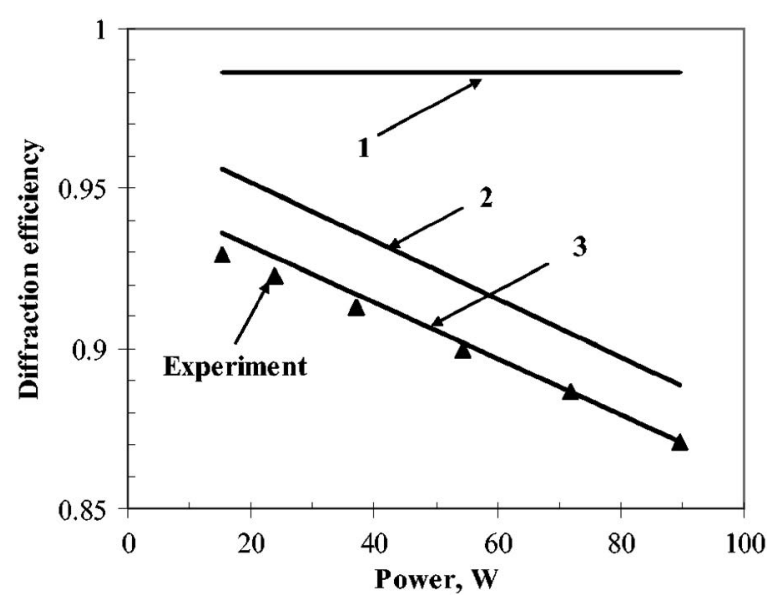

Fig. 9 Dependence of a PTR Bragg grating DE on the power of radiation with central wavelength at $1085 \mathrm{~nm}$ : triangles, experimental results; curve 1, DE calculation for monochromatic beam with 0.2-mrad-divergence; curve 2, DE calculation for planar wave with a spectral width equal to the experimental values; and curve 3 , the product of curves 1 and 2 .

VBGs. Substitution of Eqs. (22) and (25) into Eq. (26) enables modeling real beams with countable spectral width and angular divergence. For instance, if a beam has angular divergence and spectral width equal to the angular divergence and spectral selectivity of a grating with $100 \% \mathrm{DE}$ for plane monochromatic wave, its DE will be only about $35 \%$.

\section{Comparison of the Model and Experimental Results}

As noted, PTR-based VBGs typically exhibit refractive index modulation up to $1000 \mathrm{ppm}$, which is enough to secure nearly $100 \%$ efficient gratings at thicknesses less than $1 \mathrm{~mm}$ at wavelengths from the visible to near-IR regions. To prove the model experimentally, we recorded a fairly typical 1.23-mm-thick transmitting VBG with a spatial frequency of $425 \mathrm{~mm}^{-1}$ and a refractive index modulation of $420 \mathrm{ppm}$ by exposure of PTR glass to radiation from a $\mathrm{He}-\mathrm{Cd}$ laser at $325 \mathrm{~nm}$ and subsequent thermal development at $520^{\circ} \mathrm{C}$ for $2 \mathrm{~h}$. A comparative test of this grating by collimated He-Ne laser beams at 543 and $633 \mathrm{~nm}$ proved that these parameters secured calculated $100 \% \mathrm{DE}$ for a planar monochromatic wave at $1085 \mathrm{~nm}$. A 100-W cw single-transverse-mode Yb-doped fiber laser (IPG Photonics Corp., model YLR-100) with a central wavelength of $1085 \mathrm{~nm}$ was used for testing. This laser had the collimated output radiation of a 5-mm-diam Gaussian beam. It was found that this laser had a near-diffraction-limited divergence of $0.23 \mathrm{mrad}$ in the whole studied power region, while the spectral width at the $\mathrm{HW}^{-2} \mathrm{M}$ level increased from 2.7 to $4.7 \mathrm{~nm}$, when the output power rose from 15 to $100 \mathrm{~W}$.

The dependence of the DE of a PTR Bragg grating on the power of the laser at $1085 \mathrm{~nm}$ is shown in Fig. 9. One can see decreases of efficiency while power increases. Because PTR Bragg gratings have no thermally induced effects at power density levels up to $100 \mathrm{~kW} / \mathrm{cm}^{2}$ (see Refs. 16 and 31), this effect was caused by changing the laser beam parameters at different levels of emitting power. ${ }^{22}$
Based on the theoretical modeling results already described, let us evaluate how spectral width and divergence of the beam affect diffraction efficiency of this particular grating. The theoretical DE of this grating is expected to be equal to $100 \%$ for a planar monochromatic wave. The diffraction of the laser beam with 0.23 -mrad divergence on a grating with an angular selectivity of $1.6 \mathrm{mrad}$ (HWFZ) results in a decrease of DE to $98.6 \%$ (curve 1). For a planar polychromatic wave with a spectral width that increases linearly with increasing power, the calculated DE dependence on power is shown as curve 2 in Fig. 9. Curve 3, as a result of multiplication of curves 1 and 2, presents the calculated $\mathrm{DE}$ for a polychromatic divergent beam that should drop from 93.5 to $87 \%$ for beams with spectral widths of 2.7 and $4.7 \mathrm{~nm}$, respectively. The corresponding experimental data are 93 and $87 \%$ (triangles in Fig. 9). Comparison of calculated data with experimental results shows very good correspondence. Thus, the proposed model is able to describe the diffraction of polychromatic divergent beams on real PTR Bragg gratings. Another consequence of this coincidence is that the experimental PTR Bragg grating is very close to a sinusoidal uniform grating, as it was supposed in the model.

Note that this modeling can be applied to other photosensitive materials with high optical homogeneity that allow recording of sinusoidal refractive index modulation.

\section{Conclusions}

We presented the results of mathematical modeling based on Kogelnik's coupled wave theory for the diffraction of beams with a wide range of spectral and angular parameters on transmitting VBGs. This consideration is rather important for most practical applications when the spectral width and angular divergence of diffracted beams are far enough from the monochromatic/planar-wave model. The proposed model enables fast analytical calculation of angular and spectral dependencies of DE for normal and slanted gratings and could be used to design of devices based on a transmitting Bragg grating as well as testing tools for Bragg grating certification. The requirements for the parameters of gratings and laser beams for lossless Bragg diffraction were formulated. This theoretical model was compared with and found to be very close to experimental data observed for high-power Yb-fiber laser diffracting on a transmitting PTR Bragg grating. This model can be applied for all other thick Bragg gratings with sinusoidal refractive index modulation recorded in different phase photosensitive materials.

\section{Acknowledgments}

The work has been supported by Air Force Research Laboratory (AFRL) Contract No. F49620-01-1-0469. The authors thank Prof. Boris Ya. Zeldovich for fruitful discussions.

\section{References}

1. H. Kogelnik, "Coupled wave theory for thick hologram gratings," Bell Syst. Tech. J. 48, 2909-2945 (1969).

2. M. W. McCall, "Axial electromagnetic wave propagation in inhomogeneous dielectrics," Math. Comput. Modell. 34, 1483-1497 (2001).

3. M. R. Chatterjee and D. D. Reagan, "Examination of beam propagation in misaligned holographic gratings and comparison with the acousto-optic transfer function model for profiled beams," Opt. Eng. 38(7), 1113-1121 (1999).

4. T. Erdogan, "Optical add-drop multiplexer based on an asymmetric 
Bragg coupler," Opt. Commun. 157, 249-264 (1998)

5. J. Zhao, P. Yeh, M. Khoshnevisan, and I. McMichael, "Diffraction properties of vector synthetic volume index gratings," J. Opt. Soc. Am. B 17(6), 898-903 (2000)

6. R. T. B. James, C. Wah, K. Iizuka, and H. Shimotahira, "Optically tunable optical filter," Appl. Opt. 34(35), 8230-8235 (1995).

7. C. -C. Sun, M. -S. Tsaur, and B. Wang, "Self-generating high-order diffraction under Bragg mismatching in $\mathrm{LiNbO}_{3}$," Opt. Eng. 38(9), 1567-1572 (1999).

8. J. -W. An, N. Kim, and K.-W. Lee, "Volume holographic wavelength demultiplexer based on rotation multiplexing in $90^{\circ}$ geometry," Opt. Commun. 197, 247-254 (2001).

9. P. Dittrich, G. Montemezzani, and P. Gunter, "Tunable optical filter for wavelength division multiplexing using dynamic interband photorefractive gratings," Opt. Commun. 214, 363-370 (2002).

10. V. M. Petrov, S. Lichtenberg, A. V. Chamrai, J. Petter, and T. Tschudi, "Controllable Fabry-Perot interferometer based on dynamic volume holograms," Thin Solid Films 450, 178-182 (2004).

11. J. Zhou, W. Tang, and D. Liu, "Analysis of polarization properties of reflection volume holographic gratings," Opt. Commun. 196, 77-84 (2001)

12. J.-H. Chen, D.-C. Su, and J.-C. Su, "Shrinkage- and refractive-index shift-corrected volume holograms for optical interconnects," Appl. Phys. Lett. 81(8), 1387-1389 (2002).

13. J. J. Butler, M. A. Rodriguez, M. S. Malcuit, and T. W. Stone, "Polarization-sensitive holograms formed using DMP-128 photopolymer," Opt. Commun. 155, $23-27$ (1998).

14. V. A. Borgman, L. B. Glebov, N. V. Nikonorov, G. T. Petrovskii, V. V. Savvin, and A. D. Tsvetkov, "Photo-thermal refractive effect in silicate glasses," Sov. Phys. Dokl. 34, 1011-1013 (1989).

15. L. B. Glebov, N. V. Nikonorov, E. I. Panysheva, G. T. Petrovskii, V. V. Savvin, I. V. Tunimanova, and V. A. Tsekhomskii, "New ways to use photosensitive glasses for recording volume phase holograms," Opt. Spectrosc. 73, 237-241 (1992).

16. L. B. Glebov, V. I. Smirnov, C. M. Stickley, and I. V. Ciapurin, "New approach to robust optics for HEL systems," in Laser Weapons Technology III, W. E. Thompson and P. H. Merritt, Eds., Proc. SPIE 4724, 101-109 (2002).

17. M. G. Moharam and T. K. Gaylord, "Rigorous coupled-wave analysis of planar grating diffraction," J. Opt. Soc. Am. 71, 811-818 (1981).

18. D. Yevick and L. Thylen, "Analysis of gratings by the beam propagation method," J. Opt. Soc. Am. 72, 1084-1089 (1982).

19. J. Yu, D. Yevick, and D. Weidman, "A comparison of beam propagation and coupled-mode methods: application to optical fiber couplers," J. Lightwave Technol. 12, 797-802 (1994).

20. S. Ahmed and E. N. Glytsis, "Comparison of beam propagation method and rigorous coupled-wave analysis for single and multiplexed volume gratings," Appl. Opt. 35, 4426-4435 (1996).

21. M. S. Shahriar, J. Riccobono, M. Kleinschmit, and J. T. Shen, "Coherent and incoherent beam combination using thick holographic substrates," Opt. Commun. 220, 75-83 (2003).

22. I. V. Ciapurin, L. B. Glebov, and V. I. Smirnov, "Spectral combining of high-power fiber laser beams using Bragg grating in PTR glass," in Fiber Lasers: Technology, Systems, and Applications, L. N. Durvasula, Ed., Proc. SPIE 5335, 216-224 (2004).

23. I. V. Ciapurin, V. I. Smirnov, G. B. Venus, L. N. Glebova, E. V. Rotari, and L. B. Glebov, "High-power laser beam control by PTR Bragg gratings," in 2004 CLEO/IQEC and PhAST Technical Digest, CTuP54 (2004).

24. S. Yiou, F. Balembois, P. Georges, and J. Huignard, "Improvement of the spatial beam quality of laser sources with an intracavity Bragg grating," Opt. Lett. 28, 242-244 (2003)

25. Y. Zheng, X. Gao, H. Miyajima, and H. Kan, "High-brightness narrow-bandwidth high-power laser-diode array based on an externalcavity technique," Jpn. J. Appl. Phys. 43, L1299-L1301 (2004).

26. A. Dergachev, P. F. Moulton, V. I. Smirnov, and L. B. Glebov, "High power cw Tm: YLF laser with a holographic output coupler," in 2004 CLEO/IOEC and PhAST Technical Digest, CTHZ3 (2004).

27. G. B. Venus, V. I. Smirnov, and L. B. Glebov, "Spectral stabilization of laser diodes by external Bragg resonator," in 17th Annual Solid State and Diode Laser Technology Rev. (SSDLTR-2004) Technical Digest, p. 14 (2004).
28. O. M. Efimov, L. B. Glebov, L. N. Glebova, and V. I. Smirnov, "Process for production of high efficiency volume diffractive elements in photo-thermo-refractive glass," U. S. Patent No. 6,586,141 (2003).

29. O. M. Efimov, L. B. Glebov, and V. I. Smirnov, "High efficiency volume diffractive elements in photo-thermo-refractive glass," U. S. Patent No. 6,673,497 (2004).

30. L. B. Glebov, L. N. Glebova, A. P. Tirpak, D. Ehrt, B. Keinert, L. Canioni, M. Martinez-Rosas, and L. Sarger, "Refractive index measurements in photo-thermo-refractive glass," J. Non-Cryst. Solids (in press).

31. I. V. Ciapurin, L. B. Glebov, L. N. Glebova, V. I. Smirnov, and E. V. Rotari, "Incoherent combining of $100-\mathrm{W}$ Yb-fiber laser beams by PTR Bragg grating," in Advances in Fiber Lasers, L. N. Durvasula, Ed., Proc. SPIE 4974, 209-219 (2003).

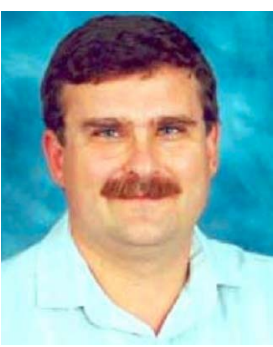

Igor V. Ciapurin is currently a research scientist with the University of Central Florida, College of Optics and CREOL. He received his MS diploma and his $\mathrm{PhD}$ degree in physics in 1984 and 1990, respectively, from the State University of Moldova, where he joined the R\&D Department in 1991 and has held different research positions. Since 2001, Dr. Ciapurin has been with CREOL in Orlando, Florida, where his research has mainly aimed to design and develop volume Bragg gratings in photothermorefractive glass for spectral beam combining of high-power lasers. He has more than 60 publications in refereed journals and conference proceedings and is a member of SPIE and OSA.

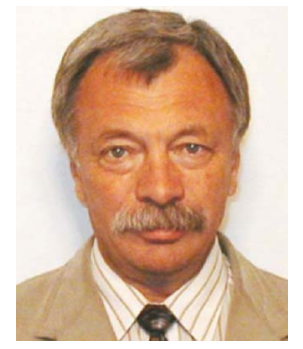

Leonid B. Glebov received his $\mathrm{PhD}$ degree in physics (with a major in optics) in 1976 from the State Optical Institute, Leningrad, Russia, with which he was affiliated until 1995, holding different positions in research and scientific management. Dr. Glebov has been a research professor with the College of Optics/CREOL at the University of Central Florida since 1995. He has published a book and more than 200 papers in scientific journals and holds 15 Russian and 3 U.S. patents. He is a member of Organizing and Program Committees for a number of international conferences. His research mainly concerns the optical properties of glasses, photosensitive glasses for hologram recording, nonlinear phenomena including laser-induced damage, and holographic optical elements.

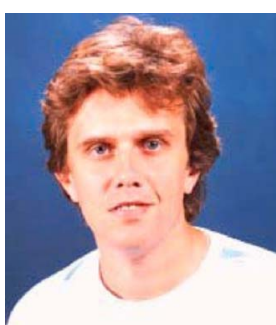

Vadim I. Smirnov is the Director of Holography and Diffractive Optics at OptiGrate. $\mathrm{He}$ is coauthor of more than 50 presentations and publications and two U.S. patents on high-efficiency diffractive elements in photothermorefractive glass. More than 7 years ago Vadim joined Dr. Glebov's research group at School of Optics/CREOL, where the technology of photothermorefractive glass was developed. His research activities include design and fabrication of volume diffractive gratings and holograms, nonlinear phenomena in optical glasses, and laser design. 